\title{
LA PEDAGOGÍA DE PAULO FREIRE Y LA FORMACIÓN CIUDADANA EN COLOMBIA: OTRA FORMA DE CONSTRUIR DEMOCRACIA DESDE LA ESCUELA
}

PAULO FREIRE'S PEDAGOGY AND CITIZENSHIP EDUCATION IN COLOMBIA: ANOTHER WAY OF BUILDING DEMOCRACY IN SCHOOLS

A PEDAGOGIA DE PAULO FREIRE E A FORMAÇÃO DE CIDADÃOS NA COLÔMBIA: OUTRA FORMA DE CONSTRUIR A DEMOCRACIA NA ESCOLA

\section{Harold Andrés Piedrahita Sánchez ${ }^{1}$} Flor Marina Hernández Herrera ${ }^{2}$

¿Cómo citar este artículo?:

Piedrahita Sánchez, H.A. y Hernández Herrera, F.M. (2021). La pedagogía de Paulo Freire y la formación ciudadana en Colombia: otra forma de construir democracia desde la escuela. Cultura Científica, 19, pp. 79 - 104. https://doi.org/10.38017/1657463X.733

1 Magíster en Filosofía, Universidad de los Andes. Especialista en Ética y Pedagogía, Fundación Universitaria Juan de Castellanos. ha.piedrahita43@gmail.com.

2 Especialista en Ética y Pedagogía, Fundación Universitaria Juan de Castellanos. hernandezherreraflormaria@yahoo.es. 


\section{RESUMEN}

Partimos de enunciar la formación ciudadana, sus objetivos y estrategias implementadas en la educación del siglo XXI. Posteriormente, analizamos el papel desempeñado por la racionalidad crítica en la pedagogía de la liberación, considerando al ser humano esencialmente en sus dimensiones dialógica, histórica y transformadora para el pensamiento de Freire, y su visión particular de la educación como realización de estas dimensiones. Luego, se describe el concepto de diálogo para este autor, y cómo este al ser desarrollado en el proceso educativo transforma las relaciones de los sujetos implicados en el acto educativo y los impulsa a considerar el contexto en el cual viven. Es importante recalcar que, esta consideración no solo busca la contemplación de la realidad sino su transformación por la acción colectiva de los actores involucrados en el proceso educativo; luego, se consideran las implicaciones que tiene el diálogo y la pedagogía de la liberación en la sociedad que se piensa y se dice democrática, por consiguiente, planteamos un estado crítico y efectivo de la igualdad y la libertad, que va más allá de las consideraciones legales y formales que pueden estar enuncia- das en un código. Además, se pretende la fundamentación y vivencia de la democracia en la construcción y práctica de este análisis crítico.

Palabras clave: crítica, pedagogía de la liberación, democracia.

\section{ABSTRACT}

This paper begins with a statement of citizenship education, its objectives and strategies implemented in the education of the 21 st century. Subsequently, we analyze the role played by critical rationality in liberation pedagogy, considering the human being essentially in its dialogic, historical and transformative dimensions for Freire's thought and his particular vision of education as the realization of these dimensions. Then, the concept of dialogue for this author is described, and how this, when developed in the educational process, transforms the relationships of the subjects involved in the educational activities and encourages them to consider the context in which they live. It is important to emphasize that, this consideration not only seeks the contemplation of reality but its transformation by the collective action of the actors involved in the educational process; then, we consider the implications that dialogue and liberation 
pedagogy have in the society that thinks and claims to be democratic, therefore, we propose a critical and effective state of equality and freedom, which goes beyond the legal and formal considerations that may be enunciated in a code. Besides, we intend the foundation and experience of democracy in the construction and practice of this critical analysis.

Keywords: critique, liberation pedagogy, democracy.

\section{RESUMO}

Começamos por enunciar a formação cidadã, seus objetivos e estratégias implementadas na educação no século XXI. Posteriormente, analisamos o papel desempenhado pela racionalidade crítica na pedagogia da libertação, considerando o ser humano essencialmente em suas dimensões dialógicas, históricas e transformadoras para o pensamento de Freire, e sua visão particular da educação como a realização dessas di- mensões. Em seguida, descreve-se o conceito de diálogo para este autor, e como este sendo desenvolvido no processo educativo transforma as relações dos sujeitos envolvidos no ato educativo e os leva a refletir sobre o contexto em que vivem. É importante ressaltar que esta consideração busca não apenas a contemplação da realidade, mas sua transformação pela ação coletiva dos atores envolvidos no processo educativo; então, são consideradas as implicações do diálogo e da pedagogia da libertação na sociedade que se pensa e dita democrática, portanto, propomos um estado crítico e efetivo de igualdade e liberdade, que vai além das considerações jurídicas e formais que podem ser declarados em um código. Além disso, pretende-se a fundamentação e experiência da democracia na construção e prática desta análise crítica.

Palavras-chave: crítica, pedagogia da libertação, democracia. 


\section{INTRODUCCIÓN}

\subsection{La formación ciudadana en Colombia propuesta por el Ministerio de Edu- cación (MEN)}

La preocupación por la formación en ciudadanía de los estudiantes es uno de los temas de actualidad que se nos presentan al interior de las instituciones educativas del país. Esta preocupación surge a raíz de diferentes problemas que enfrentamos como sociedad, una de las autoridades nacionales en formación ciudadana, el profesor Enrique Chaux lo reporta así: "tenemos de los índices de violencia más altos del mundo. Lo mismo ocurre con la corrupción y con otros problemas sociales como la inequidad $\mathrm{y}$ las discriminaciones de diverso tipo" (Chaux et al., 2004, p. 10). Si bien estos problemas nos aquejan desde hace ya bastante tiempo, los esfuerzos y formas de intervención han ganado actualidad a partir del siglo XXI, en este apartado mostraremos la génesis de esta explosividad discursiva en la educación y la innovación en las estrategias implementadas.

La formación ciudadana no se inició en el siglo XXI, en Colombia la preocupación por este tema puede rastrearse hasta los años 30 del siglo pasado con la formulación estructurada de la educación cívica (Mesa, 2008). Para nuestro propósito, nos interesa señalar la última década del siglo XX; por qué, la respuesta es triple y en varios ámbitos que afectan el mundo de la educación: la promulgación de la Constitución Política de 1991, los primeros pasos para la apertura económica y reformas neoliberales y la sanción de la ley general de educación de 1994. A lo largo de este texto, intentaremos relacionar estos eventos con las transformaciones ocurridas en la formación ciudadana al interior de la escuela.

La Constitución de 1991 es importante, ya que propone un tipo de democracia que va más allá de la representación y el voto popular, su novedad es la adición del adjetivo participativo a nuestra organización política, con el ánimo de promover que la ciudadanía se involucrara más a las decisiones concernientes al ámbito público, tal como lo muestra tanto Peralta al reconocer la importancia de la Carta Política de 1991: "la Asamblea Nacional Constituyente creó -desde el punto de vista jurídico- un marco sociopolítico democrático participativo que busca la participación ciudadana" (Peralta, 2009, p. 168). Como Torres y Díaz (2017), cuando afirman "Los años noventa inauguran un nuevo escenario sociopolítico marcado por la Constitución de 1991, cuyo principio fundamental fue la conformación de la democracia participativa en el país, para lo cual prescribió la promoción y defensa de los derechos ciudadanos como base para la construcción de un ordenamiento jurídico democrático y para la consolidación del Estado social de derecho en Colombia." (p. 48). Tenemos entonces, que la Constitución Política del 91 tenía como uno de sus grandes ob- 
jetivos, el diseño de instituciones que permitieran una mayor participación política de la ciudadanía.

En cuanto a las reformas económicas de finales del siglo $X X$, podemos afirmar que se orientaban principalmente a la reducción de la burocracia estatal, la ampliación de la racionalidad económica a diversas esferas sociales y la apertura del mercado nacional a los productos importados,

\begin{abstract}
A partir de la década de los noventa se hace evidente en Colombia el predominio del proyecto político neoliberal y su encuadramiento en las determinaciones impuestas por el proceso de globalización capitalista. Entre sus efectos más notorios encontramos el desmonte del Estado social o de bienestar y su reducción a un Estado "mínimo, eficiente y competitivo" organizado en la lógica económica y de acuerdo con las reglas del mercado. (Torres y Díaz, 2017, p. 49).
\end{abstract}

Por último, la Ley 115 de 1994 llamada Ley General de Educación, para nuestro interés en este artículo promueve la democratización de la escuela a través de la formación "para la participación y la organización infantil (objetivo específico), otorgándole a la participación estudiantil un papel esencial que garantiza a los estudiantes ser tomados en cuenta en los ámbitos decisivos de cada institución educativa" (Peralta, 2009, p. 169).

Es dentro de este ambiente que, el Ministerio de Educación Nacional diseña e implementa una nueva manera de educación ciudadana por medio de las competencias ciu- dadanas y sus estándares básicos. A continuación, explicaremos los que consideramos los puntos esenciales del programa.

Para el profesor Chaux, investigador y promotor de las competencias ciudadanas, uno de los motivos para emprender esta ventura pedagógica, es denunciar una falta de democracia en la cotidianeidad de los colombianos. Este autor lo denuncia al manifestar que la gran brecha entre la realidad y los ideales de la constitución, "lo que ocurre en la cotidianidad de la vida de millones de colombianos y colombianas está todavía lejos de lo que se espera de una verdadera democracia y de lo que propone nuestra Constitución Política." (Chaux et al., 2004, p. 10). Una de las causas que identifica el autor, es "el énfasis casi exclusivo en los conocimientos. Es muy común, por ejemplo, que la formación ciudadana se limite al aprendizaje del contenido de la Constitución Política Nacional. Este aprendizaje es importante, pero no es suficiente para la acción ciudadana." (Chaux et al., 2004, p. 14) Es decir, en la educación ciudadana las intervenciones de los docentes se enfocan en transmitir conocimientos y nada más, por lo tanto, no hay maneras de poner a prueba estos conocimientos, no hay una intención en promover la acción ciudadana de los estudiantes; al contrario, la formación ciudadana propuesta por el MEN "propicia la reflexión intencional sobre las finalidades y límites de la esfera política, implica el desarrollo de la capacidad deliberativa y la preparación

\footnotetext{
"Lo que ocurre en la cotidianidad de la vida de millones de colombianos y colombianas está todavía lejos de lo que se espera de una verdadera democracia y de lo que propone nuestra Constitución Política."
} 
La Revolución

Educativa, reflejada en la noción de competencia, propone que lo importante no es sólo conocer, sino también saber hacer. Se trata, entonces, de que las personas puedan usar sus capacidades de manera flexible para enfrentar problemas nuevos de la vida cotidiana para la participación responsable en procesos sociales y políticos" (Ruíz y Chaux, 2005, p. 19).

Esta preparación para la deliberación y acción ciudadana, pretende deshacerse de "una pedagogía cuya prioridad era la memorización de contenidos, [aproximándose a una pedagogía] que promueve la construcción de conocimiento con sentido. Es decir, un conocimiento que adquiera significación a partir de una razón de ser, de una utilidad en la vida práctica" (Ministerio de Educación Nacional y Fondo de Población de las Naciones Unidas, 2006).

Por consiguiente, se desalientan las explicaciones magistrales por parte del profesor y una actitud pasiva de los estudiantes, y más bien, se promueve el interés y la participación de estos últimos por medio de preguntas relacionadas con sus conocimientos previos, sus experiencias y su cotidianeidad. En este nuevo tipo de educación, no importa tanto la cantidad de información que el estudiante pueda almacenar y reproducir cuando se le solicite; por el contrario, lo que se busca es que los estudiantes "puedan usar sus capacidades de manera flexible para enfrentar problemas nuevos de la vida cotidiana" (Ministerio de Educación Nacional, 2004).

Este uso flexible de las capacidades propuesto por el MEN (2004), parte de la introducción en el mundo educativo de la noción de competencia, considerando que "ser competente [...] significa saber y saber hacer. La competencia implica poder usar el conocimiento en la realización de acciones o productos" (p. 7). Por lo tanto, la novedad de este enfoque educativo radica en el protagonismo dado a las actividades desarrolladas por el estudiante, es decir, se privilegia la capacidad de hacer del estudiante, "La Revolución Educativa, reflejada en la noción de competencia, propone que lo importante no es sólo conocer, sino también saber hacer. Se trata, entonces, de que las personas puedan usar sus capacidades de manera flexible para enfrentar problemas nuevos de la vida cotidiana." (Ministerio de Educación Nacional, 2004).

En este enfoque de acción ciudadana, las competencias ciudadanas son definidas como:

los conocimientos y habilidades que permiten que el ciudadano actúe de manera constructiva en la sociedad democrática. [...], las competencias ciudadanas se evidencian en la práctica, en lo que las personas hacen. La acción ciudadana (ejercida de manera autónoma y no por imposición por parte de otros) es el objetivo fundamental de la formación ciudadana. (Chaux, 2004, p. 20)

Entonces, tenemos que las competencias ciudadanas son una serie de saberes y destrezas que se ejecutan en nuestras relaciones sociales, en nuestra vida en comunidad, pero teniendo en cuenta que el objetivo es lograr esa vida social sin agresión y respetando la diversidad.

Dentro de las competencias ciudadanas, tenemos la siguiente clasificación: conocimientos, cognitivas, 
emocionales, comunicativas e integradoras. Esta tipología pretende dar respuesta a las dimensiones del aprendizaje que mencionamos anteriormente: saber y saber hacer en contextos cotidianos y flexibles.

Relevante para nuestro propósito, es analizar la definición de una de las competencias cognitivas de la ciudadanía, el pensamiento crítico, concebido como "la capacidad para cuestionar y evaluar la validez de cualquier creencia, afirmación o fuente de información. De esta manera podemos cuestionar lo que ocurre en la sociedad e identificar cómo la realidad podría ser distinta a cómo es actualmente." (Chaux et al., 2004, p. 22). Esta competencia tiene como fundamento, el análisis lógico de contenidos cognitivos que podamos tener, por ejemplo: la democracia es buena, la paz es un bien deseable, los conflictos se resuelven a golpes; estos contenidos son analizados a través de la confrontación con los hechos, experiencias e ideales que podemos compartir, con el objetivo de validarlos y promoverlos, en caso de que sean fines loables o, por el contrario, transformar nuestra cotidianeidad y acercarnos más a la propuesta de democracia participativa pregonada por la constitución: "Una sociedad que quiere ser realmente democrática requiere de la participación activa y crítica de todos. Esto implica que todos sus miembros deben poder estar involucrados en la construcción de acuerdos y en la toma de decisiones" (Chaux et al., 2004, p .19), en la cual "La búsqueda de consensos debe reflejar las distintas posiciones y puntos de vista, y debe darse a través del diálogo directo entre los involucrados." (Chaux et al., 2004 p. 20).

Por otra parte, en la actualidad educativa se promueve un interés cada vez más acuciante de evaluar el impacto de las políticas educativas. En cuanto a la formación ciudadana, tenemos que, junto a la concepción e implementación de la noción de competencias ciudadanas, aparece en el discurso educativo la noción de estándar, la cual es definida en plural como "criterios claros y públicos que permiten establecer cuáles son los niveles básicos de calidad de la educación a los que tienen derecho los niños y niñas de todas las regiones de nuestro país" (Ministerio de Educación Nacional, 2004). En otras palabras, tenemos que la educación debe ser de calidad, y esta calidad es apreciada o medida a través de criterios objetivos que permiten comparar el desempeño de los estudiantes con una norma establecida como aceptable.

Dentro de la formación por competencias ciudadanas, el enfoque evaluativo ha sido preponderante desde el inicio de la aplicación del programa, tal como Torres y Díaz (2017) lo afirman:

a partir de 1996, el gobierno nacional adelantó una serie de pruebas masivas para medir el estado de la ciudadanía en el contexto escolar, lo cual constituyó una apuesta congruente con la tendencia neoliberal impuesta en la década de los noventa, que determinó el diseño de las políticas educativas a partir de la definición y medición de indicadores de eficiencia, eficacia y calidad (p. 48).

\author{
"Una sociedad \\ que quiere ser \\ realmente \\ democrática \\ requiere de la \\ participación acti- \\ va y crítica \\ de todos. Esto \\ implica que todos \\ sus miembros \\ deben poder \\ estar involucrados \\ en la construcción \\ de acuerdos y \\ en la toma de \\ decisiones"
}


Esta evaluación tiene el propósito explícito de determinar la utilidad o funcionalidad de la estrategia, con el ánimo de identificar las debilidades para mejorarlas y reforzar las actividades pedagógicas exitosas al resolver los problemas de discriminación, violencia y agresión. El instrumento más utilizado para determinar los aciertos del programa, es la evaluación de competencias ciudadanas aplicada de manera censal por el Instituto Colombiano para la Evaluación de la Educación (ICFES). Esta evaluación se hace utilizando un instrumento psicométrico de preguntas con única respuesta, cuyos temas van desde "conocimientos sobre el Estado (ciudadano informado), actitudes positivas frente a la democracia (ciudadano virtuoso), y etapa del desarrollo moral en la cual se encuentran los niños (ciudadano racional)." (Cortés, 2009, p. 110), y pretende "saber cuáles [maneras de formación ciudadana] son más efectivas para lograr lo que buscamos y si estamos realmente logrando avances." (Chaux et al., 2004, p. 18).

Antes de pasar al análisis de la propuesta de Freire, consideramos oportuno resumir lo expuesto hasta este punto. La educación ciudadana ha sido una preocupación estatal de vieja data, si nos atenemos a la formalización de la educación cívica, podemos encontrar un hito en la república liberal de la década de 1930. Otro de los hitos lo encontramos al final del siglo $\mathrm{XX}$, con la Ley General de Educación, la Constitución Política del 91 y la apertura económica, fenómenos que influ- yeron en la revolución educativa concebida a partir de las nociones de competencia, ciudadanía activa y evaluación de la educación.

\subsection{Racionalidad crítica y edu- cación}

Como pueblo latinoamericano, no desconocemos que, desde nuestros orígenes, hemos sido una población colonizada, y por ende todos nuestros aspectos culturales han venido transcurriendo, en cada tiempo, con este sello. Si nos detenemos en el aspecto educativo, elemento de nuestro estudio y análisis, veremos en los distintos cuestionamientos dicha situación, vista críticamente a través los planteamientos de Paulo Freire.

En la historia de la educación colombiana, se conocen varios modelos educativos, los primeros centrados en lo que denominaron disciplina como obediencia a la norma establecida por los adultos y la sumisión que ésta imprimía, de modelos de enseñanza a modelos de aprendizaje, pero siempre modelos traídos de fuera, aplicados a nuestra realidad colombiana, donde no se han detenido a mirar esta "realidad” que difiere significativamente con el lugar de donde provienen estos modelos.

Dichos modelos educativos adaptados, se encargaron de construir esa enseñanza tradicional y opresora que critica Freire, a la que denomina "Educación Bancaria", dado que su propósito es depositar conocimientos; y si bien es cierto, nuestra educación colombiana no ha im- 
partido otra cosa que ese estilo de formación en sus 200 años de experiencia que conocemos. De igual forma, estos estilos educativos han venido generando estudiantes pasivos, que se han "llenado" de conocimientos, que han desarrollado prodigiosamente su capacidad memorística. Así mismo, de esta educación "bancaria" somos nosotros los actuales maestros, el producto; generaciones dóciles a la autoridad, al "jefe", al "rector", que es el que "sabe" porque tiene "muchos conocimientos" y estudios, puede que hasta en el exterior.

Como contraparte a esta visión técnica, tenemos la racionalidad crítica, interés emancipatorio y concienciación en la pedagogía de la liberación. Estos aparecen como fruto del debate creado al interior de las ciencias sociales, principalmente en la primera mitad del siglo $X X$; fundamentado en la definición del objeto de dichas ciencias y el método apropiado para estudiarlo, se abrieron diferentes corrientes de pensamiento que problematizan la relación entre sujeto y objeto, y método de investigación.

Dentro de esta discusión, en primera instancia, enunciamos la posición del positivismo lógico defendida por Popper (1978), quien plantea en su ponencia de la lógica de las ciencias sociales, que el punto de partida de éstas es una contradicción entre el conocimiento y los hechos, y que dicha contradicción conlleva a teorizar y generar problemas teóricos para los cuales se ensayan diferentes soluciones que pretenden remediar la contradic- ción inicial, mejor dicho lograr la armonía entre teoría y hechos; sin embargo, en este punto es necesario recalcar que la solución a un problema determinado se da al encontrar la teoría que mejor explique los hechos observados.

Ahora bien, para saber cuál ensayo de solución es mejor, Popper apela a la crítica, pero ésta es considerada únicamente en el terreno de la lógica deductiva. Además, Vasco (1994) manifiesta que la ciencia como expresión de la racionalidad positivista tiene el interés principal de "predecir lo que va a pasar para poder controlar si es posible; ese interés que busca el dominio de la naturaleza $[. .$.$] es el interés técnico,$ [...], es el interés por la predicción y el control”.(p. 17) Es decir, las ciencias sociales consideradas desde esta perspectiva teórica pretenden encontrar leyes que explican la situación actual de los fenómenos sociales, los cuales son el objeto de estudio, y sus variaciones para así poder predecir resultados y de esta manera controlar el devenir del objeto de estudio.

Dentro de la pedagogía, podemos apreciar que esta posición epistémica pretende tratar la formación de los estudiantes como un problema que deber ser resuelto a través de la aplicación de teorías y técnicas que maximizan y predicen los resultados, reducen los esfuerzos; al respecto, Diniz-Pereira y Soares (2019) consideran que "Os modelos mais difundidos de formação de professores são aqueles orientados pela racionalidade técnica. De acordo com esse modelo, também 
conhecido como a epistemologia positivista da prática, [...], o professor é visto como um técnico, um especialista que rigorosamente põe em prática as regras científicas e/ ou pedagógicas." (p. 2-3). En este sentido, la formación ciudadana de los estudiantes estará regida por teorías y técnicas diseñadas por expertos en este tipo de conocimientos, que luego son aplicadas por los docentes.

Por el contrario, la posición crítico social defendida entre otros por Adorno (1978), afirma que "la contradicción no tiene porqué ser, como Popper supone al menos aquí [en La lógica de las ciencias sociales], una contradicción meramente "aparente" entre el sujeto y el objeto e imputable exclusivamente al sujeto en calidad de insuficiencia de juicio" (p. 32). Para los pensadores críticos, la debilidad del enfoque positivista radica en que para éste el conocimiento al ser armonía entre teoría y hechos, no tiene ningún motivo para proponer alternativas a una realidad dada, como lo dice Giroux (1992) "en la visión tecnocrática de la racionalidad positivista la cuestión de la oposición entre la realidad del mundo y su ideal se reduce a la tarea metodológica de recolectar y clasificar hechos" (p. 11). En cambio, la ciencia crítica social busca abrir el espacio para otras alternativas para la realidad, tal y como lo enuncia Vasco (1994).

Podríamos decir que hay un tipo de ciencia, [...], que busca develar aquellas cadenas veladas $u$ ocultas con las cuales nos encontramos atados en nuestra praxis histórica y busca encontrar la manera de romperlas, de liberarnos de ellas. No tiene un interés meramente especulativo, simplemente de ver que es lo que hay ahí, describirlo, explicarlo y comprenderlo, sino de proporcionar armas teóricas para romper esas cadenas que ha mostrado que existen. (p. 21)

De hecho, la ciencia comprometida con esta transformación de la realidad, va más allá de proporcionar armas teóricas y busca una praxis que permita superar la opresión y enajenación, el interés de este enfoque de trabajo en las ciencias sociales es definido como "interés emancipatorio" y "está caracterizado; [...], por develar las dependencias en el sentido de quitar el velo que oculta las ataduras y por consiguiente romperlas" (Vasco, 1994, p. 22). Es más, para Diniz-Pereira y Soares (2019) "Na racionalidade crítica, a educação é "historicamente localizada" - ela acontece contra um pano de fundo sócio-histórico e projeta uma visão do tipo de futuro que nós esperamos construir -, uma atividade social - com consequências sociais, não apenas uma questão de desenvolvimento individual" (p. 4); es decir, que la educación desde el punto de vista crítico tiene un localización espacio-temporal precisa que nos interpela para transformar nuestra realidad y superar la opresión, la importancia de esta localización espacio-temporal dentro de la racionalidad crítica de Freire se debe a que una de las características principales de ser humano es "ser en situación: "situado y fechado»: Un ser engarzado en el espacio y en el tiempo que su conciencia intencionada capta y tras- 
ciende." (Freire y Torres, 1980, p. 52). En otras palabras, la compresión que tenemos sobre nosotros mismos se debe al tiempo y lugar en que habitamos. Desde esta racionalidad crítica, los docentes son los encargados de construir problemas que muestran la dimensión política de las situaciones vividas por los estudiantes y su entorno, vale la pena recalcar que esta visión problematizadora de los docentes no tiene un tinte técnico; desde este punto de vista, los problemas se conciben, lo dijimos anteriormente, como domesticación de la realidad y su manipulación para que se adecue a las teorías que permiten explicarla y controlarla: "Os modelos técnicos têm uma concepção instrumental sobre o levantamento de problemas; os práticos têm uma perspectiva mais interpretativa $\mathrm{e}$ os modelos críticos têm uma visão política explícita sobre o assunto." (Diniz-Pereira y Soares, 2019, p. 5).

Entonces, considerando lo anteriormente enunciado, pensamos que para la pedagogía crítica la teoría no es un bien producido por unos y consumido por otros; al contrario, la teoría es una construcción solidaria realizada por los miembros de una comunidad por medio de la praxis y con el objetivo también solidario de un beneficio comunitario, y por consiguiente seguimos la concepción que presenta Giroux (1992)

[En la pedagogía crítica] la interacción entre la teoría y la práctica no se encuentra en el punto en que los "radicales" suministran las prescripciones y los padres de familia, trabajadores y oprimidos los reciben los utilizan; en vez de eso, se encuentran en el punto en que estos diferentes grupos se reagrupan y plantean la pregunta fundamental de cómo ellos pueden esclarecer a cada uno de los otros y como a través de tal intercambio puede emerger un modo de práctica en el que todos los grupos se pueden beneficiar. (p. 11)

Ahora bien, la opresión a la cual nos referimos se puede dar en "la abrumadora necesidad de producir y consumir el despilfarro; la necesidad de un trabajo embrutecedor cuando ha dejado de ser una necesidad; la necesidad de modos de descanso que alivian y prolongan ese embrutecimiento" (Marcuse, 1985, p. 37). Entonces, al aparecer estas necesidades (en un principio únicamente económicas, pero que en la actualidad implican un estilo de vida en particular) y las formas de suplirlas hasta puntos insospechados e individualizantes, toman la forma del velo que se alza y oculta otras posibilidades de realización para el ser humano que las prescritas por los opresores con el ánimo de controlar a las masas e impedir su preocupación en los asuntos públicos.

Dicho de otro modo, encontramos que la opresión del ser humano se da en tanto se imponen unas necesidades y la forma de satisfacerlas, por ejemplo, en nuestro caso en los círculos de la cultura desarrollados aparecían frases, tales como "tengo que comprar el último Iphone o sino que dirán" o "darle a mis hijos las cosas que yo nunca tuve". Esta expresión de deseos desenfrenados que 
son transformados en necesidades, es para Freire (1974) una de las características del hombre alienado u oprimido a quien le importa más parecer que ser.

Por lo tanto, vemos que la opresión tiene como consecuencia una especie de servidumbre: de vivir según los dictados de lo que se considera bueno, y de esta manera la vida humana se reduce a una actividad y a un trabajo alienante. Sin embargo, y para no perder las esperanzas el pensamiento crítico, propone que "toda liberación depende la toma de conciencia de la servidumbre" (Marcuse, 1985, p. 37), ahora bien, la toma de conciencia significa una apertura a la comprensión de las estructuras sociales como formas de dominación. Es decir, si queremos transformar nuestra sociedad debemos entender cómo se conforman las estructuras y divisiones de clase como el primer paso para tomar conciencia.

Por lo tanto, es necesario resaltar que, para Freire (2009), la toma de conciencia tiene tres categorías que son:

$$
\begin{aligned}
& \text { la intransitividad de la conciencia } \\
& \text { que es una conciencia cerrada y } \\
& \text { centrada en las necesidades vita- } \\
& \text { les, la transitividad ingenua que } \\
& \text { es gregaria, polémica, con pobre } \\
& \text { argumentación y fija en el tiempo } \\
& \text { con la idea de que el pasado fue } \\
& \text { mejor y la transitividad crítica que } \\
& \text { está volcada a la responsabilidad } \\
& \text { social y política. (p. } 54)
\end{aligned}
$$

En otras palabras, una conciencia cerrada está determinada por el ultraindividalismo que se manifiesta en frases como "hay que ser avión"
- "papaya puesta, papaya partida"; una conciencia ingenua que se caracteriza por la inactividad, por la preocupación únicamente enfocada en el suplir necesidades fisiológicas básicas (Freire, 2009), por el cruzarse de brazos esperando que nada pase o que todo empeore o por esperar que las transformaciones necesarias se darán por arte de magia, en "la transitividad ingenua, [...], se caracteriza, entre otros aspectos, por la simplicidad en la interpretación de los problemas; por la tendencia a juzgar que el tiempo mejor fue el tiempo pasado; [...]; por la fragilidad en la argumentación; por un fuerte tenor emocional; por la práctica no propiamente del diálogo sino de la polémica..." (Freire, 2009, p.54); por último, la conciencia crítica es esencialmente histórica, esto es, considera que la realidad es producto del trabajo de los hombres en conjunto no sólo de algunos de ellos, por lo tanto, es una conciencia que se sabe responsable social y políticamente, que profundiza en su comprensión del pasado, y además que el futuro no está predeterminado sino que se actualiza en el presente y la praxis le da forma. Freire (1974) condensa esa relación entre historia, conciencia y pedagogía, así:

la educación crítica afirma que los hombres son seres que se superan $[. .$.$] seres para los cuales la$ inmovilidad representa una amenaza fatal, para los cuales mirar al pasado no debe ser sino un medio de comprender más claramente quiénes son y qué son para poder construir el horizonte con más sabiduría. (p. 52) 
Entonces, para lograr la emergencia de la conciencia crítica Freire (1974), propone que:

Era indispensable para obrar la transformación esencial del paso de una comprensión mágica e ingenua a una comprensión crítica, hacer recorrer al hombre sencillo un camino a través del cual tomara conciencia de:

1. La existencia de dos mundos: naturaleza y cultura

2. El papel activo del hombre en la realidad

3. El papel de mediación que juega la naturaleza en las relaciones y en las comunicaciones entre los hombres

4. La cultura como resultado de su trabajo, de su esfuerzo creador y recreador

5. El papel del hombre, que es de sujeto y no de simple objeto. (p. 39)

Ahora, para lograr este tránsito desde la conciencia cerrada hasta la crítica, Freire (2009) propone una pedagogía que elimina de raíz las relaciones autoritarias en las que ya no existe "escuela" ni "profesor", sino "círculos de cultura" y "coordinador", cuya tarea esencial es el diálogo, por consiguiente es la negación de la revelación de la verdad y de la dicotomía sujeto-objeto.

Por lo tanto, el círculo de la cultura es la unidad de enseñanza en la pedagogía de la liberación en oposición a la escuela autoritaria. Esta oposición se da en el círculo de la cultura cuando se presentan relaciones democráticas fundamentadas en el diálogo; por el contrario, en la escuela autoritaria el diálogo es proscrito y se alienta el monólogo del educador.
Luego, es necesario dialogar pero sobre algo en concreto y ese algo son situaciones desafiantes basadas en las características del mundo cultural y natural, en el carácter activo del hombre y sobre todo de temas que "tendrán que ser existenciales para tales grupos [poblacionales]" (Freire, 1974, p. 78); es decir, que tengan importancia, que susciten un problema para la cotidianidad de los integrantes del círculo de la cultura, y es este carácter problemático de las situaciones es la génesis de los temas generadores, los cuales buscan una toma de posición y de acción por los integrantes del círculo de la cultura ante dicha situación desafiante.

En consecuencia, la propuesta de la pedagogía de la liberación se basa en un encuentro comunitario en el que el trabajo es fruto de la cooperación entre coordinador y educandos, en el cual "la naturaleza formadora de la docencia no podría reducirse al puro proceso técnico y mecánico de transferir conocimientos, enfatiza la exigencia ético-democrática del respeto al pensamiento, gustos, recelos, deseos curiosidad de los educandos" (Freire, 1993, p. 102), así vemos que para la pedagogía crítica el problema de la didáctica pierde relevancia debido a que el propósito es abrir un espacio para la formación de sujeto y, por lo tanto, se opta por el diálogo como herramienta para dicha formación.

Vemos ahora, que el papel de la teoría en la pedagogía de la liberación no es la explicación ni el control de hechos sociales ni mucho menos su transmisión de alguien que la co-

\author{
“La naturaleza \\ formadora de la \\ docencia no \\ podría reducirse \\ al puro proceso \\ técnico y mecánico \\ de transferir \\ conocimientos, \\ enfatiza la \\ exigencia \\ ético-democrática \\ del respeto al \\ pensamiento, \\ gustos, recelos, \\ deseos curiosidad \\ de los educandos"
}




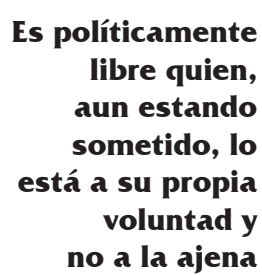

Es políticamente libre quien, aun estando sometido, lo voluntad y no a la ajena noce, para este caso el docente, a alguien que la desconoce, el estudiante; sino la comprensión crítica de los hechos sociales.

Entonces, si este es el papel del educador, este debe estar articulado con el de un investigador que no puede usar metodologías que estén sesgadas hacia el positivismo y su pretensión de universalidad y objetividad; por el contrario, la concienciación no es un hecho universal, es un hecho histórico que debe ser desarrollado por sujetos comprometidos, y, por tal razón, pensamos que como Freire (1993) señala "una misma comprensión de la práctica educativa, una misma metodología de trabajo no opera necesariamente de forma idéntica en contextos diferentes. [...]. Por eso insisto en que las experiencias no pueden ser trasplantadas" (p. 53).

En otras palabras, en las intervenciones de corte positivista es posible y deseable universalizar métodos y teorías por medio de un trabajo investigativo en el cual es menester cuantificar conductas, objetivos investigativos, entre otros; es decir, establecer una línea base y compararla con una línea en la que se muestran los resultados fruto de la intervención investigativa, para determinar si esta tuvo el efecto deseado; todo esto con ánimo de transformar la realidad por medio de la técnica. Por el contrario, para la investigación crítica, el objetivo es lograr una comprensión emancipadora de la realidad tanto para el investigador como para el colaborador e investigado, para que de manera solidaria sea posible transformar la realidad.

\subsection{Diálogo, conciencia crítica y democracia}

Para comenzar este apartado, es necesario enunciar alguna definición de la democracia. Siguiendo el pensamiento de algunos autores, tenemos que la democracia es la búsqueda de la autodeterminación, es decir, la búsqueda de la libertad, tal como Kelsen (1934) lo expresa al manifestar que "es políticamente libre quien, aun estando sometido, lo está a su propia voluntad y no a la ajena" (p. 17). Sin embargo, este ideal visto como no someterse a una voluntad extraña, no significa simplemente una anulación de lo social, más bien, es necesario un cambio en el significado de esta. Dicho cambio comienza al considerar la existencia del Estado, es decir, la existencia de la autoridad; si debemos ser gobernados, entonces lo deseable es que nos gobernemos nosotros mismos. Freire (2009), siguiendo a Tocqueville, también considera fundamental para la democracia la condición de autogobierno. Incluso, el asesor del MEN considera que en los regímenes democráticos el pueblo tiene el poder fundamental de decidir su destino y, por tal razón, utiliza el adjetivo de participativa para calificar a la democracia en la cual los ciudadanos son capaces de cambiar los acuerdos construidos por sus representantes,

en una democracia participativa los representantes no tienen el poder último sobre las decisiones. Es decir, ese poder recae en los representados, quienes tienen distintos mecanismos 
para ejercer el control como, por ejemplo, el voto programático (votar por un programa que los candidatos se comprometen a cumplir), la transparencia en la ejecución (que los representados puedan conocer en detalle la actuación de sus representantes), o la revocatoria del mandato (que los electores puedan exigir el cambio de representantes si consideran que los elegidos no están representando bien sus posiciones e intereses). (Chaux et al., 2004, pp. 19-20)

Por su parte, Foucault (2009) plantea que

la definición, digamos, morfológica, de la democracia en los textos teóricos de Platón, Aristóteles, etc., es relativamente fácil de obtener, $[\ldots]$. Es el gobierno del demos, es decir el conjunto de ciudadanos. [Para] la caracterización de aquello en que consiste [la democracia] se recurre a unas cuantas nociones como, por ejemplo, la de la eleutheria (libertad), otra característica que se invoca es la isegoria, la igualdad de la palabra, la posibilidad que tiene todo individuo, con tal de formar parte, desde luego, del demos, de formar parte de los ciudadanos, de disfrutar el acceso a la palabra, [...], emitir opinión en el transcurso de una discusión de un debate. (p. 162)

Como resumen de estas posiciones, podemos afirmar, en primer lugar, que la democracia es el gobierno del pueblo; segundo, la caracterización de pueblo como conjunto de ciudadanos que gozan de ciertos derechos como la libertad, es decir, no estar dominados ni subyugados por otro y la igualdad en la oportunidad de pronunciar la palabra en público, mejor dicho, de participar en el espacio público. Entonces, se asume que la palabra es importantísima en la democracia, ya que es el medio para expresar las necesidades, sueños, expectativas acerca del bien común, quien es el ser; es decir, es una palabra que surge de la conciencia crítica.

Ahora bien, estos aspectos no sólo nos importan en el marco de una participación que en la sociedad se adquiere con la mayoría de edad; al contrario, interesa expandir el horizonte de la democracia a todas las esferas de la vida humana, tal y como Freire (2009) señala

La democracia que antes que forma política es forma de vida se caracteriza sobre todo por la gran dosis de transitividad de conciencia en el comportamiento humano, transitividad que no nace y no se desarrolla salvo bajo ciertas condiciones, en las que el hombre se lance al debate, al examen de sus problemas y de los problemas comunes, en las que el hombre partícipe. (p. 76)

Para tal efecto, se analizó la libertad y la igualdad del discurso desde la perspectiva de la pedagogía de la liberación.

\subsubsection{Libertad}

Para el pensamiento crítico y la pedagogía de la liberación, la libertad tiene dos connotaciones; por un lado, es el punto de partida de la praxis; y, por otro lado, es la meta a la cual esa praxis transformadora anhela llegar.

Es punto de partida en la medida en que para Freire es condición fundamental del ser humano, ya que no 
es posible obligar a alguien a dialogar, por mucho es posible arrancar una confesión, una repetición de un discurso alienante. Al contrario, y como se vivió anteriormente, el diálogo implica el respeto y la pluralidad.

Si la nuestra [práctica educativa] es una opción progresista, sustantivamente democrática, debemos, respetando el derecho que tienen los educandos de optar también y de aprender a optar, para lo que precisan de libertad, testimoniarles la libertad con la que optamos (o los obstáculos que tuvimos para hacerlo) y jamás intentar, subrepticiamente o no, imponerles nuestra idea. (Freire, 1993, p. 104)

De esta manera, es posible considerar que la libertad se da en un ambiente social y que debe ser elaborada constantemente, no es algo dado, ni regalado ni mucho menos impulsa a la ultra individualización.

En la sociedad, se parte de considerar que, por un lado, ya presenta formas en las que la libertad es entendida como la disponibilidad de acceder a hacer y tener lo que se quiera, es decir "hacer lo que se da la gana”; o por la ilusión de pensar por sí mismo cuando lo cierto es que se piensa como los demás debido a un proceso de homogenización social. Por otra parte, quien no cuenta con dicha disponibilidad, se encuentra en una situación de dependencia principalmente económica, y desde luego una situación de dominación en las demás esferas de la vida humana.
Se tienen, en consecuencia, dos condiciones que amenazan la libertad y que surgen de un poder que, en el primer caso, es enfrentado con violencia; $y$, en el segundo con sumisión, y que son problematizados por la teoría crítica de la siguiente manera: para la supuesta libertad "la amplitud de la selección abierta a un individuo no es factor decisivo para determinar el grado de libertad humana” (Marcuse, 1985, p. 37).

En consecuencia, uno de los propósitos de la pedagogía crítica con respecto a la democracia es analizar las condiciones en las que se encuentra la sociedad y posteriormente proponer alternativas construidas solidaria y dialógicamente, que modifiquen esas condiciones actuales; es decir, partir de una libertad formal, analizarla y transitar hacia una libertad crítica y una participación efectiva.

Segundo, para la vida en medio de la dependencia económica Freire (2009) afirma que

$$
\begin{aligned}
& \text { El gran peligro del asistencialismo } \\
& \text { está en la violencia de su antidia- } \\
& \text { logo, que imponiendo al hombre } \\
& \text { mutismo y pasividad no le ofrece } \\
& \text { condiciones especiales de desa- } \\
& \text { rrollo o apertura de su concien- } \\
& \text { cia, que en las democracias au- } \\
& \text { ténticas debe ser cada vez más } \\
& \text { crítica. (p. } 50 \text { ) }
\end{aligned}
$$

Entonces, el asistencialismo al poner condiciones a la población para poder calificar para recibir las ayudas propuestas, impide las condiciones necesarias para que los seres humanos se apropien de sus condiciones históricas y tengan conciencia de su carácter de suje- 
tos en la transformación del mundo, "a industrialização vem intensificando formas 'assistencializadoras' de ação, já analisadas por nós e que , 'domesticando' o homem, lhe dificultam a promoção da consciência ingênua à crítica ..." (Haddad, 2019, p. 5); por ejemplo, en este caso, los programas de asistencia social y promoción de salud se enfocan en el cumplimiento de determinadas tareas y requisitos por parte de la población como que tengan determinado puntaje en el SISBÉN, asistencia a controles de salud, permanencia en el sistema educativo, entre otras; requisitos y tareas que son impuestos sin una articulación histórica y dialógica con la población. Por el contrario, son impuestos por un saber antidialógico que se revela en la palabra oficial de un funcionario, palabra que por ser oficial obviamente no puede ser discutida o contextualizada simplemente obedecida.

Por otra parte, la democracia se sustenta en pluralidad de puntos de vista, diversidad de opiniones, pero con libertad e igualdad en las opciones de acceder al espacio público para sustentar esas opiniones y puntos de vista. En esta democracia, "el hombre radical en su opción no niega el derecho a otro de optar. No pretende imponer su opción, dialoga sobre ella. Está convencido de su acierto, pero respeta en otro el derecho de juzgarse también dueño de la verdad; intenta convencer y convertir, pero no oprime a su oponente" (Freire, 2009, p.41). Por consiguiente, y como Zuleta (1995) expresa "la democracia no es el de- recho de la mayoría, es el derecho del otro a diferir iesa es la democracia que vale la pena alcanzar!" (p. 47); para el pensamiento crítico, la democracia es libertad de decir pero con responsabilidad de lo que se dice, además de respeto por la diferencia. Por consiguiente, la voluntad popular no se logra simplemente por la adición de individuos que imponen su punto de vista en razón a su mayor número como sucede en la democracia liberal; por el contrario, para la teoría crítica tanto el uso de la razón o conciencia crítica (libertad), como el respeto expresado en la protección a las minorías (participación) son los fundamentos de la democracia.

\subsubsection{Participación ciudadana y esfera pública}

De lo anteriormente expuesto, se extrae que es difícil considerar una situación de igualdad en el uso de la palabra como herramienta para la construcción del bien común; en el primer caso, la apatía como consecuencia del individualismo acérrimo.

Un analfabeta político -independientemente de que sepa leer y escribir- es aquel que tiene una concepción ingenua de las relaciones de la humanidad con el mundo. Este tipo de persona tiene una visión ingenua de la realidad social absolutamente determinada (Freire, 1990, p. 116).

En el segundo caso, oprimidos por el miedo y mudos debido a la situación de dominación, "el hombre trágicamente asustado, que teme la convivencia auténtica y que duda de

\author{
Un analfabeta \\ político \\ -independiente- \\ mente de que \\ sepa leer y \\ escribir- es aquel \\ que tiene una \\ concepción \\ ingenua de las \\ relaciones de la \\ humanidad con \\ el mundo
}


“La democracia es frágil.

Su fragilidad procede de que es difícil aceptar el grado de angustia que significa pensar por uno mismo" sus posibilidades, al mismo tiempo se inclina a un gregarismo" (Freire, 2009, pp. 34-35).

El analfabetismo y apatía política es resultado de la conciencia cerrada o ingenua que considera el mundo como algo ya hecho e inmodificable, ya en nuestra práctica educativa escuchamos respuestas de este tipo "la sociedad es asi", "pero siempre ha sido asi”"; por el contrario, la transitividad crítica de la conciencia posibilita la comprensión de la relación dialéctica entre hombre y mundo, y el carácter existencialmente transformador del ser humano.

En consecuencia, para la sociedad es más fácil callarse y adherirse a otros que indiquen de manera técnica y antidialógica el camino que se debe seguir y eliminar ese sentimiento de angustia que es propio de la democracia, "la democracia es frágil. Su fragilidad procede de que es difícil aceptar el grado de angustia que significa pensar por uno mismo" (Zuleta, 1995, p. 77); en efecto, es necesario aceptar la responsabilidad de los discursos que se puede pronunciar en el espacio público para buscar el bien común.

Además, las élites que nunca se han interesado en la emancipación de sus congéneres, consideran que la "democracia se deteriora cuando las clases populares están demasiado presentes [...] denunciando la fealdad del mundo y anunciando un mundo más bello" (Freire, 1993, p. 58); es decir, sus intereses particulares de dominación se fundamentan en mantener la situación en un estado de mutismo de las masas, $y$, si por algún motivo se presenta un cambio, este es controlado y asimilado para mantener el status quo.

Entonces, para las elites, la democracia es participación, pero vedada para aquellos que no cumplan ciertas características: "que no sean gente de bien”, que no sepan hablar, que digan en público lo que no se debe decir, o bien que sean ignorantes.

Por el contrario, el pensamiento crítico busca la participación de todos los miembros de la comunidad y de esta manera posibilitar la transformación de la realidad, ya que dicha "[participación] adecuada es aquella que rompe la relación de dominación y dependencia entre gobernantes y gobernados y la transforma en simétrica como debería ser" (Fals Borda, 1987, p. 38). Esta simetría se da en el respeto y la igualdad efectiva para participar en el cuidado de lo público, en la posibilidad de que exista pluralidad de discursos en un espacio público incluyente, en el cual esta pluralidad de voces lucha por la transformación.

Por consiguiente, el reto es construir una unidad en medio de la diversidad, y se cree que la participación fundamentada en el diálogo es definitiva para esta construcción de unidad en cualquier dimensión de la vida humana, y además que si se opta por la pedagogía crítica y se compromete con la emancipación propia y de sus congéneres, debe considerar "la participación, en cuanto ejercicio de voz, de tener 
voz, de injerir, de decidir en ciertos niveles de poder, en cuanto ejercicio del derecho ciudadano, se halla en relación con la práctica progresista” (Freire, 1993, p. 70).

Entonces, para el pensamiento crítico es fundamental que, en la democracia, la participación por medio del ejercicio de la palabra sea efectiva y no simplemente en el ejercicio de un sufragio. Esto no significa la recomendación de una democracia directa; por el contrario, es el reclamo de situar la toma de decisiones en "[la esfera pública que] constituye el ámbito sociocultural de la sociedad como un terreno importante en la batalla ideológica por la apropiación del estado, la economía y la transformación de la vida diaria” (Giroux, 1992, p. 294) a través del diálogo comprometido.

Pero, en la situación actual, la palabra popular como fundamento de legitimación en la esfera pública, ha sido opacada por la tecnocracia administrativa que, además de callar al pueblo, lo oprime y aísla de la lucha por el bien común; en oposición a estas prácticas actuales, las investigaciones críticas y entre ellas la pedagogía de la liberación, proponen una aperturidad a la diversidad y al trabajo comunitario tal como lo dice Fals Borda (1987)

Estas redes pluralistas se van expandiendo en el espacio geopolítico de la ciudad o el campo para promover entre todos, la ayuda mutua, unidad popular, defensa de la vida, investigación social, concientización y educación de adultos. Proceden de las bases hacia arriba y de la periferia al centro, en lo cual contradicen a la ortodoxia y rutinas partidistas (p. 25).

En consecuencia, para el pensamiento crítico, el diálogo y la afirmación práctica y real de las características de la democracia, se despliegan en la esfera pública para promover la emancipación. Allí, de manera especial, la educación

Tiene un vínculo con la creación de esferas públicas alternativas. [...]. Se refiere a formas de aprendizaje y acción basadas en un compromiso por eliminar formas de opresión, [...]. Se refiere a la crítica y a la reconstrucción de experiencias sociales en formas nuevas de interacción comunicativa. (Giroux, 1992, p. 298)

Ahora bien, no hay que creer ingenuamente que, con la creación de esas esferas públicas alternativas, se ha solucionado el problema de la inexperiencia democrática y la opresión, solamente se ha abierto el espacio para que la tarea sea ocupar este lugar con la participación efectiva de la comunidad, y fruto de esta ocupación seguramente se abrirán nuevos caminos y vivencias de la democracia, tal como lo expone Freire (2009) "la posición transitivamente crítica implica un regreso a la verdadera matriz de la democracia." (p. 55).

\section{CONCLUSIONES}

El esfuerzo expositivo de este trabajo, se orientó a mostrar las características tanto de la propuesta de formación en competencias ciudadanas promovida por el MEN, tanto de la pedagogía de la liberación

\author{
“La esfera pública \\ que constituye \\ el ámbito \\ sociocultural de \\ la sociedad \\ como un terreno \\ importante en \\ la batalla \\ ideológica por \\ la apropiación \\ del estado, \\ la economía y \\ la transformación \\ de la vida diaria"
}


de Freire; ahora, es momento de contraponer estas dos propuestas. Teniendo en mente este objetivo, este último apartado discurrirá de manera sucinta entre los orígenes, noción de crítica, concepción y realidad de la democracia y fines.

Como vimos anteriormente, la formación por competencias ciudadanas nace en un ambiente de promoción de la democracia, reformas educativas y económicas. Con relación a este ambiente, podemos afirmar que la nueva Constitución se percibe como evento que produce un giro en las prácticas de la educación ciudadana, al promover la participación de la ciudadanía en una variedad de aspectos públicos, tales como consultas, referendos, revocatorias de mandato. Dentro de ese ánimo de expandir la ciudadanía, el MEN concibe que fortalecer el desarrollo de las competencias ciudadanas

es tomar la decisión de hacer democracia en el país, de formar unos ciudadanos y ciudadanas comprometidos, respetuosos de las diferencias y defensores del bien común. [...] las ciudadanas y los ciudadanos formados de esta manera podrán utilizar la Constitución Política como guía hacia la sociedad deseada y medio para realizarla, y ejercer como ciudadanos y ciudadanas competentes, una ciudadanía que conduzca a la ampliación de la democracia participativa como medio para la realización plena del Estado Social de Derecho y el logro de la paz como consecuencia lógica de dicha realización (Ministerio de Educación Nacional y Fondo de Población de las Naciones Unidas, 2006, p. 30).
Entonces, podemos apreciar cómo esta nueva forma de educación ciudadana se articula con la propuesta constitucional, sirviendo como herramienta que debe transformar la sociedad y acercarnos a los ideales propuestos en la Carta Magna de 1991, en tanto que los estudiantes se formarán como ciudadanos activos, respetuosos de la diversidad y participativos. Así aparece entonces, que la ciudadanía implica unas relaciones flexibles en un mundo cotidiano, donde no basta con tener unos conocimientos específicos; además de estos, es necesaria una acción que permita tanto transformar al individuo como a la sociedad en la cual actúa.

Ahora bien, para llegar a este loable objetivo, es necesario que en la escuela "todos los adultos involucrados en la educación nos propongamos promover y construir ambientes democráticos reales" (Ministerio de Educación Nacional, 2004, p. 9), en los cuales la participación de los estudiantes les permita experimentar que su voz es tenida en cuenta en la construcción de los manuales de convivencia, en los protocolos de resolución de conflictos, en el gobierno escolar, entre otras esferas de acción posible.

Ante tanta maravilla, podríamos manifestar que hemos superado el silencio impuesto a los oprimidos que Freire denunciaba en las sociedades antidemocráticas en las que

el pueblo es un enfermo al cual se deben aplicar remedios. Y su enfermedad es precisamente tener voz y participación. Cada vez que intente expresarse libre- 
mente y pretenda participar, es señal de que continúa enfermo, necesitando entonces mis "remedios". La salud para esta extraña democracia es el silencio del pueblo, su quietud. (Freire, 2009, pp. 47-48).

Pareciera que, como comunidad, hemos abierto el espacio para participar y actuar en la esfera pública, incluso hasta la niñez y la juventud pueden participar activamente en la escuela. Sin embargo, el pedagogo brasileño nos advierte que, para tener una participación auténtica, es necesaria "la práctica del diálogo y no de la polémica. [...] la receptividad de lo nuevo, no sólo por nuevo, y [...] la no-negación de lo viejo, sólo por viejo" (Freire, 2009, pp. 54-55)

Tenemos entonces, por lo menos dos puntos a los que debemos prestar atención: no dejarnos embaucar por la novedad de las prácticas que podrían ocultar formas insospechadas de opresión; la transitividad crítica de la conciencia nos alerta ante soluciones mágicas, como que lo nuevo es bueno simplemente en virtud de su novedad; y poder diferenciar claramente el diálogo de la polémica, ésta última es la herramienta del sectarismo, que "tiene una matriz preponderantemente emocional y acrítica, es arrogante, antidialogal y por eso anticomunicativa, [...]. El sectario nada crea porque no ama. No respeta la opción de los otros. Pretende imponer la suya -que no es opción sino fanatismo- a todos." (Freire, 2009, p.42).

Como podemos apreciar en la polémica, tenemos una guerra entre dos bandos: el de la verdad y los iluminados contra el de la mentira y el engaño; en tanto es una guerra, existe una fuerte carga emocional marcada por el odio y la animadversión, que no permite valorar críticamente los prejuicios y encontrar puntos de encuentro; aparece la dicotomía vencer o ser vencido, dominar o ser dominado. Por consiguiente, nos encontramos una vez más con la posibilidad de la opresión y de negarle a los otros su dimensión de sujeto, para considerarlos simplemente como cosas que deben ser manipuladas para satisfacer las necesidades que nos aquejan, tenemos entonces una afirmación de la identidad del yo o del nosotros, siguiendo un patrón individualista y de exclusión (yo soy/nosotros somos en tanto usted/ es no son).

En tanto que "na perspectiva freireana, a participação requer diálogo, que por sua vez é o encontro respeitoso de reflexão mútua." (Streck et al., 2017, pp. 15-16). Por lo tanto, podemos advertir que el proceso de participación que en la actualidad vemos desarrollarse en la vida escolar, parece hacer realidad la propuesta freiriana del pensamiento crítico; sin embargo, consideramos que la participación como fin en sí misma debe acompañarse de la concienciación crítica para que, de esta manera, la participación no sea un ejercicio meramente formal o de confrontación de intereses, sino de la búsqueda de la emancipación para todos los que se comprometen en un diálogo auténtico. 
Por otra parte, la lógica neoliberal impulsó la reducción del tamaño del estado, desregularización de relaciones comerciales, y expansión de la racionalidad económica a esferas diferentes al intercambio de bienes; una muestra de estas expansiones se muestra en el mundo educativo, en tanto que "la educación deja de ser un derecho humano, y la escuela deja de ser un lugar de vigencia de lo público, para convertirse en "servicio transable", abriendo las puertas a una plena organización mercantil de la educación.» (Torres y Díaz, 2017, p. 49).

Una de las consecuencias de esta propuesta económica, es que «el Estado de hoy experimenta la injerencia de grandes corporaciones y organizaciones de origen privado y la correspondiente delegación de competencias públicas a sectores no estatales. Es una tendencia mundial, en la que el "achicamiento" del Estado constituye una urgencia para los capitales transnacionales." (Peralta, 2009, p.170). Otra de las consecuencias del modelo neoliberal, es un exacerbado ánimo que defiende la idea de la libertad del individuo de escoger entre una multitud de opciones, de fomentar

\footnotetext{
"a igualdade de oportunidades" para os "cidadãos" (também recorrentemente concebidos como "consumidores") competirem no mercado global. O fato de que a "oportunidade", a liberdade e a escolha estejam diminuindo cada vez mais em outras esferas de vida da maioria dos cidadãos-consumidores, sujeitas às demandas das necessidades das grandes corporações (Diniz-Pereira y Soares, 2019, p.7).
}

Tenemos entonces que la libertad de escoger se da, pero dentro de una esfera de opciones dominadas por la mano invisible del mercado y la rentabilidad, en la cual los individuos dejan de ser considerados ciudadanos con responsabilidades políticas, sociales, estéticas, entre otras, y se convierten en agentes económicos interesados en maximizar sus ganancias. Por lo tanto, se "impone una subjetividad individualista, consumista, conformista, pasiva e intolerante. Cada quien busque salvarse por su cuenta y aplastando a los demás si es necesario, promoviendo la resignación ante esta realidad que aparece como la única posible y no tiene sentido querer cambiarla." (Jara, 2019, p.3).

Dentro de esa lógica económica, aparecen las competencias ciudadanas como una estrategia que permite a los estudiantes desarrollar las habilidades necesarias para superar la discriminación, gestionar los conflictos de manera pacífica y participar en el gobierno escolar. Otra de las novedades educativas, es la tendencia a evaluar (casi de manera obsesiva) los aprendizajes de los estudiantes para determinar la utilidad y el impacto de la formación en competencias ciudadanas. Sin embargo, este afán evaluativo a través de pruebas censales, traiciona el espíritu práctico de la propuesta del MEN, al determinar "el grado de desarrollo cognitivo-político de las niñas, los niños y los jóvenes, derivando conclusiones generales que, en muchos casos, desconocen la complejidad de la 
formación social y política de los mismos" (Torres y Díaz, 2017, p. 62). Al respecto y siguiendo al pensamiento de Freire, podemos decir que la masificación y estandarización de las pruebas nos impone una visión deformada tanto de la realidad como de los sujetos de la evaluación, transformado la primera en una entidad susceptible de la manipulación técnica operada desde las teorías científicas orientadas por el afán de predicción y control, y a los segundos en engranajes anónimos que pueden ser moldeados también por la técnica y la teoría; realidad expuesta por nuestro pedagogo de manera magistral, al afirmar que

\begin{abstract}
Una de las grandes - si no la mayor- tragedias del hombre moderno es que hoy, dominado por la fuerza de los mitos y dirigido por la publicidad organizada, ideológica o no, renuncia cada vez más, sin saberlo, a su capacidad de decidir. Está siendo expulsado de la órbita de las decisiones. El hombre simple no capta las tareas propias de su época, le son presentadas por una élite que las interpreta y se las entrega en forma de recela, de prescripción a ser seguida. Y cuando juzga que se salva siguiendo estas prescripciones, se ahoga en el anonimato, índice de la masificación, sin esperanza y sin fe, domesticado y acomodado: ya no es sujeto. Se rebaja a ser puro objeto. (Freire, 2009, p.33)
\end{abstract}

Dentro de los fines planteados por el programa de formación en competencias ciudadanas, tenemos la promoción de comportamientos individuales que faciliten la convivencia pacífica, la participación responsable en los procesos de- mocráticos y el respeto de las diferencias (Ministerio de Educación Nacional, 2004). Con respecto a la participación democrática considerada como autodeterminación de los pueblos, será necesario precisar la actual contradicción entre la lógica neoliberal y la autonomía estatal, sobre todo en naciones en desarrollo que se encuentran bajo la tutoría de organismos internacionales que influyen en los estados a través de préstamos bancarios. Al respecto, hay coincidencia entre varios de los autores consultados en que, por ejemplo, el Banco Mundial influye en políticas educativas de acuerdo con intereses económicos, por ejemplo, tenemos tres afirmaciones que muestran este fenómeno "el Banco Mundial está jugando un papel hegemónico en el rediseño de las políticas educativas en América Latina. Esto no tiene que ver con la claridad o poder de convicción de sus ideas, sino con su poder económico" (Peralta, 2009, p. 170); coincidentemente con el anterior autor, "o Banco Mundial (BM) tem sido um dos mais importantes veículos de divulgação da racionalidade técnica e científica em reformas educacionais" (Diniz-Pereira y Soares, 2019, p. 3). De esta injerencia del Banco Mundial tenemos que las políticas educativas son diseñadas siguiendo lineamientos foráneos y que responden a intereses particulares: "es claro que la incidencia de los organismos internacionales en la formulación de las políticas educativas de los países latinoamericanos o en vía de desarrollo, aparece como uno de los síntomas característicos del contenido de las

\author{
El hombre \\ simple no capta \\ las tareas propias \\ de su época, \\ le son presentadas \\ por una élite que \\ las interpreta y \\ se las entrega en \\ forma de recela, \\ de prescripción \\ a ser seguida.
}


relaciones entre las instituciones educativas o el sistema educativo en general y el entorno social, político y económico en el cual dicho sistema se desenvuelve." (Peralta, 2009, p. 171).

Estas políticas educativas que idealmente promueven la participación democrática en la práctica, dificultan la consecución de ese objetivo al privilegiar la competencia mercantil en el servicio educativo por medio de la evaluación y la adjudicación de presupuesto y beneficios, con base en indicadores de gestión académica y resultados en pruebas estandarizadas (Peralta, 2009).
Finalmente y siguiendo la conclusión de Torres y Díaz (2017), tenemos una fuerte contradicción con "la coexistencia de una Constitución Política que se abre a las posibilidades de la democracia participativa concebida como forma de vida ciudadana y la adopción de un modelo neoliberal que gradualmente niega los ideales de la democracia, limita los derechos y restringe los espacios para el ejercicio de la ciudadanía" (p. 48). Tenemos en la pedagogía de Freire, herramientas interesantes que nos pueden ayudar en la comprensión de esta contradicción actual y nos animan a actuar para superarla. 


\section{REFERENCIAS}

Adorno, T. (1978). Sobre la lógica de las ciencias sociales. Coponencia. En La Lógica de las ciencias sociales (pp. 29-46). Editorial Grijalbo S.A.

Chaux, E., Lleras, J., y Velásquez, A. M. (Eds.). (2004). Competencias ciudadanas: De los estándares al aula, una propuesta de integración a las áreas académicas (1. ed). Ministerio de Educación Nacional / CESO / Ediciones Uniandes.

Cortés, R. A. C. (2009). Educación ciudadana en Colombia: Dos coordenadas para un posible análisis. Educación y ciudad, 16, 105-118.

Diniz-Pereira, J. E., y Soares, L. J. G. (2019). Formação de educadoras/es, diversidade e compromisso social. Educação em Revista, 35, e217314. https://doi.org/10.1590/0102-4698217314

Fals Borda, O. (1987). Ciencia propia y colonialismo intelectual. Los nuevos rumbos. Carlos Valencia Editores.

Foucault, M. (2009). El gobierno de sí y de los otros. Curso en el College de France (1982-1983). Fondo de Cultura Económica de Argentina.

Freire, P. (1974). Concientización: Teoría y práctica de la liberación. Ediciones Búsqueda.

Freire, P. (1990). La naturaleza política de la educación. Paidós.

Freire, P. (1993). Política y educación. Editorial Laboratorio Educativo.

Freire, P. (2009). Educación como práctica de libertad. Siglo XXI Editores.

Giroux, H. (1992). Teoría y resistencia en la educación; una pedagogía para la oposición. Siglo XXI Editores.

Haddad, S. (2019). Política, educação e atualidade do pensamento freiriano. Educação em Revista, 35, e214048. https://doi.org/10.1590/01024698214048

Jara, O. (2019). Palavra abierta - educación popular, contexto latinoamericano y el legado de Paulo Freire. Educação em Revista, 35, e215005. https:// doi.org/10.1590/0102-4698215005

Kelsen, H. (1934). Esencia y valor de la democracia. Labor.

Marcuse, H. (1985). El hombre unidimensional. Planeta de Agostini.

Mesa, A. (2008). La formación ciudadana en Colombia. Revista Uni-pluriversidad, 8(3 Suplemento). https://revistas.udea.edu.co/index.php/unip/article/view/1814 
Ministerio de Educación Nacional. (2004). Formar para la ciudadanía sí es posible. Ministerio de Educación Nacional: http://www.mineducacion.gov. co/1621/articles-75768_archivo_pdf.pdf

Ministerio de Educación Nacional y Fondo de Población de las Naciones Unidas. (2006). Proyecto piloto de educación para la sexualidad y construcción de ciudadanía: hacia la formación de una política pública. Imprenta Nacional de Colombia.

Peralta, B. (2009). La formación ciudadana en el sistema educativo de colombia: ¿una mirada reactiva o transformadora? Eleuthera, 3, 165-178.

Ruíz, A., y Chaux, E. (2005). La Formación de Competencias Ciudadanas $\left(1^{\circ}\right)$. Asociación colombiana de facultades de educación - Ascofade.

Streck, D. R., Pitano, S. de C., y Moretti, C. Z. (2017). Educar pela participação, democratizar o poder: o legado freireano na gestão pública. Educação em Revista, 33(0). https://doi.org/10.1590/0102-4698167880

Torres, J., y Díaz, A. (2017). Las vías de la educación ciudadana en Colombia. Revista Folios, 47. https://doi.org/10.17227/01234870.21 folios47.64

Vasco, C. E. (1994). Tres estilos de trabajo en las ciencias sociales. CINEP.

Zuleta, E. (1995). Educación y democracia, un campo de combate. Fundación Estanislao Zuleta. 